cerebellum, spinal cord, and brainstem. ${ }^{[2]}$ The unusual locations include leptomeninges and sellar-sphenoid sinus. $^{[4-6]}$ Supratentorial location accounts for 4 to $13 \%$ of the cases. ${ }^{[2]}$ However, hemangioblastomas of the lateral ventricle are extremely rare and only 5 cases have been reported in the English literature till 2001. ${ }^{[2]}$ We report a unique case of a lateral ventricular hemangioblastoma.

A 30-year-old male presented with a progressive holocranial headache of six months duration. The neurological examination was normal. Magnetic resonance imaging (MRI) of the brain showed a $3 \times 2 \mathrm{~cm}$ mass in the left lateral ventricle, which was hypointense on T1-weighted images, hyperintense on T2-weighted as well as fl uid attenuated inversion recovery (FLAIR) images. The tumor showed brilliant contrast enhancement [Figures 1-3]. The patient had a left-sided parietal craniotomy and through a trans-sulcal approach through the superior parietal

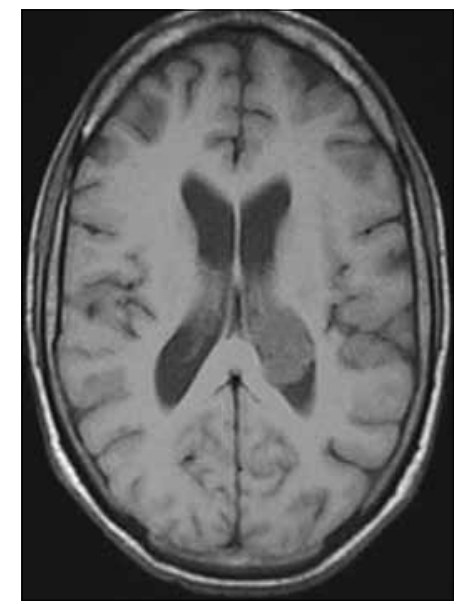

Figure 1: T1-weighted axial magnetic resonance imaging scan of the patient showing a mass in the body and atrium of the left lateral ventricle which is hypointense compared to the normal white matter

\title{
Hemangioblastoma of the lateral ventricle
}

Sir,

Hemangioblastomas are the most frequent vascular tumor of the central nervous system $(\mathrm{CNS})^{[1]}$ and can be associated with von Hippel-Lindau (VHL) disease in 3 to $38 \%$ of the cases as a major manifestation. ${ }^{[2]}$ These tumors are predominantly found in the

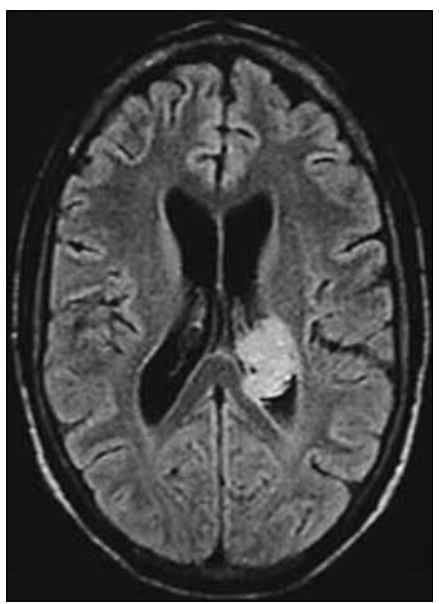

Figure 2: T2-weighted fluid attenuated inversion recovery axial scan of the patient showing a mass in the body and atrium of the left lateral ventricle which is hyperintense compared to the normal white matter 
lobule the lesion was excised. At operation the lesion was brownish red, soft to firm, highly vascular, and was adherent to the ventricular ependyma as well as the choroid plexus. An immediate post-operative computed tomography (CT) scan showed complete excision of lesion [Figure 4]. Post-operatively, the patient had transient sensory aphasia, which recovered fully in a 1 month. A histopathological examination showed tissue composed of a fi ne network of blood spaces separated by numerous polygonal stromal cells, with lighty stained cytoplasm [Figure 5]. Immunohistochemical staining showed a strong immunopositivity in several stromal cells for vimentin, epithelial membrane antigen (EMA), and neuron specifi c enolase (NSE) - typical of a hemangioblastoma. The patient was investigated for other features of von Hippel-Lindau syndrome but none were found.

Hemangioblastomas of the CNS are infrequent and they account for $2 \%$ of primary CNS tumors. ${ }^{[1,3]}$ The most common location is the posterior fossa, cerebellar hemisphere accounting for $83 \% .{ }^{[3]}$ Supratentorial location is rare, sporadic hemangioblastomas $4 \%$ and VHL disease $13 \% .^{[2]}$ The lateral ventricular location is extremely unusual, only four symptomatic cases $^{[7-10]}$ and one incidental postmortem finding ${ }^{[8]}$ have been described. All the four lateral ventricle hemangioblastomas reported till date had been in patients with VHL disease. ${ }^{[7-10]}$ In our patient we could not find any evidence for VHL disease. However, it is prudent to investigate patients with lateral ventricular hemangioblastoma for VHL disease. The size of the lateral ventricle hemangioblastomas in all the four documented cases has been greater than the size of the hemangioblastoma in other locations. This may partly be related to the fact that the volume of the lateral ventricle can accommodate a reasonable size mass lesion and any mass lesion in the lateral ventricle has to grow to a sufficient size to produce symptoms. In other locations cystic component of hemangioblastoma has been the contributing factor to the size of the tumor, whereas all the lateral ventricle hemangioblastomas including our case were solid. ${ }^{[7-10]}$

Hemangioblastoma of the lateral ventricle is associated with a better prognosis than its counterpart in the third ventricle. Diehl and Symon were able to achieve complete resection of the tumor. ${ }^{[11]}$ Our experience was also similar. We were able to achieve good control of the feeding vessels from the choroids plexus and the wall of the lateral ventricle, thus enabling in toto resection of the tumor. Post-operative dysphasia/ mutism is an infrequent and usually transient complication of hemangioblastoma surgery. In our case, it was probably related to the proximity of the tumor to the posterolateral region of the thalamus and/or surgeryrelated edema and/or ischemia in

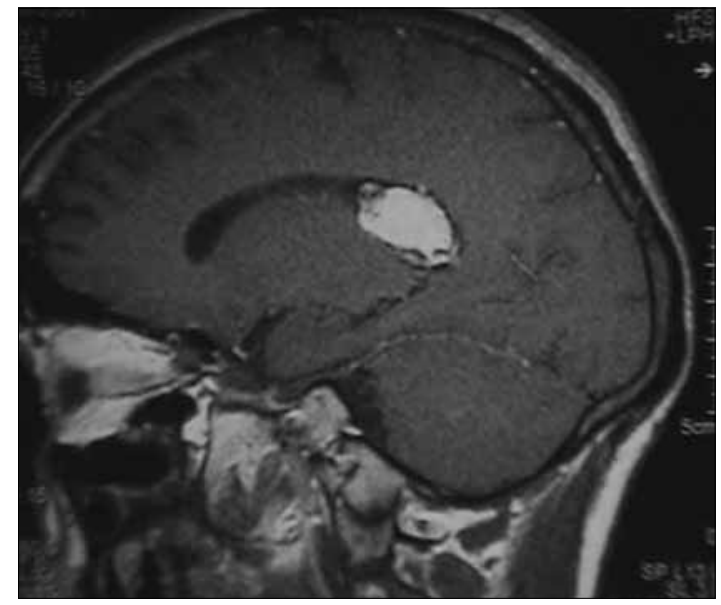

Figure 3: T1-weighted sagittal magnetic resonance imaging scan of the patient showing brilliant contrast enhancement of the lateral ventricular mass

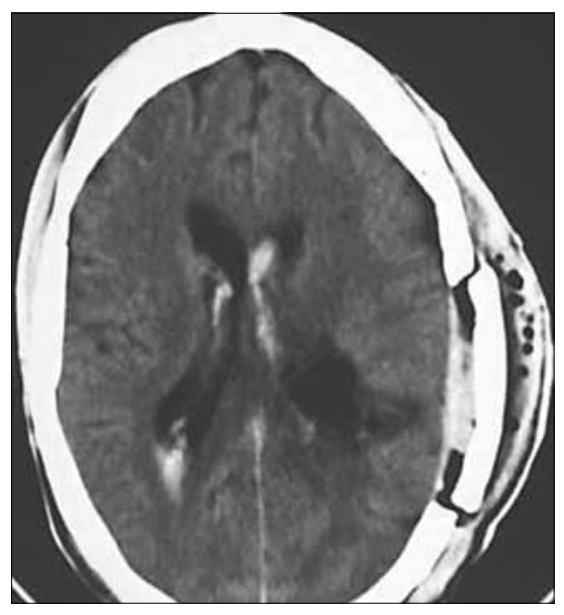

Figure 4: Post-operative axial head computed tomography scan of the patient showing complete excision of the left ventricular lesion

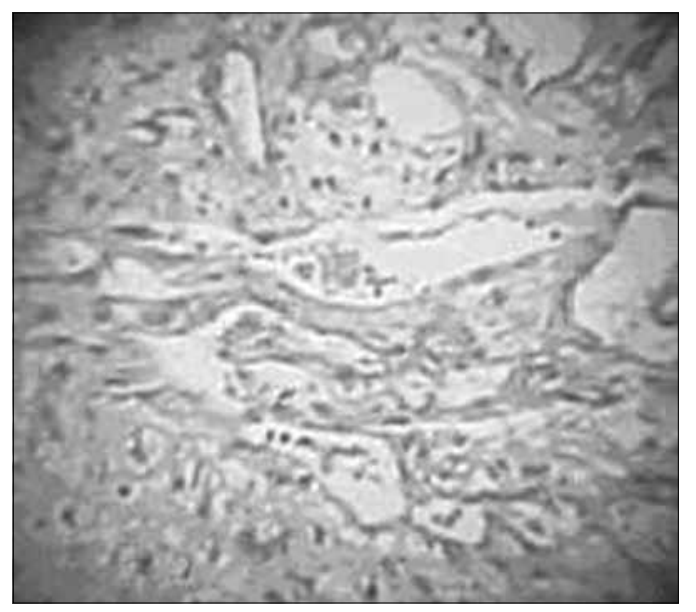

Figure 5: Histopathological photograph showing a tissue composed of a fine network of blood spaces separated by numerous polygonal stromal cells, with lightly stained cytoplasm 
the parietal region. Its incidence can be reduced by delineating the speech area by functional MRI preoperatively ${ }^{[12]}$ or by using intraoperative cortical mapping. ${ }^{[13]}$

Ramandeep Singh Jaggi,
Ishwar Chandra Premsagar, Abhishek
Department of Neurosurgery, Dr. R.M.L. Hospital, Baba Kharak
Singh Marg, New Delhi - 110 001, India.
E-mail: rsjaggi2000@yahoo.com
PMID: 19934579
DOI: 10.4103/0028-3886.57797

\section{References}

1. Strugar J, Criscuolo G. Primary intracranial vascular tumors. In: Vecht C, editor. Handbook of clinical neurology. Neuro-Oncology, 1997. Part II, pp 269-86.

2. Conway J, Chou D, Clatterbuck R, Brem H, Long DM, Rigamonti D. Hemangioblastomas of the central nervous system in von Hippel-Linday syndrome and sporadic disease. Neurosurgery 2001;48:55-63.

3. Weil RJ, Vortmeyer AO, Zhuang Z, Pack SD, Theodore N, Erickson RK, et al. Clinical and molecular analysis of disseminated hemangioblastomatosis of the central nervous system in patients without von Hippel- Lindau disease. J Neurosurg 2002;96:775-87.

4. Reyns N, Assaker R, Louis E, Lejeune JP. Leptomeningeal hemangioblastomatosis in a case of von Hippel-Lindau disease: Case report. Neurosurgery 2003;52:1212-6.

5. Sajadi A, Tribolet N. Unusual locations of hemangioblastomas. J Neurosurg 2002;97:727.

6. Kachhara R, Nair S, Radhakrishnan VV. Sellar-sphenoid sinus hemangioblastoma: Case report. Surg Neurol 1998;50:461-4.

7. Prieto R, Roda JM. Hemangioblastoma of the lateral ventricle: Case report and review of the literature. Neurocirugia (Astur) 2005;16:58-62.

8. Vecehi B, Patrassi G. Angioretriculoma del plessi corioidei, con "aree di Gamna”. Schweiz Med Wochenschr 1935;65:242-6.

9. Ho YS, Plets C, Goffin J, Dom R. Hemangio-blastoma of the lateral ventricle. Surg Neurol 1990;33:407-12.

10. Murakami H, Toya S, Otani M, Sato S, Ohiera T, Takenaka N. A case of concomitant posterior fossa and supratentorial hemangioblastomas. No Shinkei Geka 1985;13:175-9.

11. Diehl P, Symon L. Supratentorial intraventricular hemangioblastoma: Case report and review of literature. Surg Neurol 1981;15:435-43

12. Hall WA, Kim P, Truwit CL. Functional magnetic resonance imagingguided brain tumor resection. Top Magn Reson Imaging 2009; 19:205-12.

13. Brell M, Conesa G, Acebes JJ. Intraoperative cortical mapping in the surgical resection of low-grade gliomas located in eloquent areas. Neurocirugia (Astur) 2003;14:491-503.

Accepted on 10-2-2009 